\title{
A Framework for Describing Theoretical Perspectives: Overview and Application to the Work System Perspective
}

\author{
Steven Alter \\ University of San Francisco \\ alter@usfca.edu
}

\begin{abstract}
This paper summarizes a framework for describing theoretical perspectives (an FDTP) and uses it to describe the work system perspective (WSP) in a way that integrates ideas that often had been discussed in isolation from each other. It also illustrates how the FDTP can support application of the WSP to the idea of digital transformation (DT). It discusses the general value of the FDTP along with potential applications.
\end{abstract}

\section{Introduction}

This paper contributes to an ongoing inquiry at HICSS about "knowing what we know: where to now?" [1] that has been extended into "informing research: where to now?" [2]. Its contribution to that inquiry assumes that those questions call for clear understandings of theoretical perspectives and clear distinctions between different theoretical perspectives. Those concerns are important in the IS discipline because many IS research publications claim to use a specific theoretical perspective without defining that perspective carefully, without explaining boundaries of its relevance, and without explaining how it is related to non-obvious generalizations about the subject matter. Typical examples are claiming to use a systems perspective or a service perspective but not defining what those perspectives mean, instead merely citing a number of existing sources that seem to represent their use even if different sources interpret them differently.

Goal and organization. This paper provides a broadly applicable approach for addressing those issues. It identifies an FDTP, illustrates its application to a specific theoretical perspective (the WSP), and further illustrates its application to DT, a currently important concept that can be discussed by using the WSP within the WSP's limitations. This paper pursues the following goal related to a framework for describing theoretical perspectives:
Demonstrate the value of a carefully articulated FDTP by identifying an FDTP, showing that it can be used to organize key ideas in an existing theoretical perspective and also showing that it can be used to organize discussion of a currently important topic.

HICSS restrictions on paper length necessitate using a condensed approach that only summarizes many points and limits the number of citations. The sections of the paper proceed as follows: 2) provide background by defining theoretical perspective for current purposes and summarizing the development of the FDTP, 3) summarize the FDTP, 4) apply the FDTP to the WSP in general, 5) apply the FDTP to the specific concept of DT consistent with the purpose of proposing a DT success theory (DTST), and 6) discuss implications and conclusions related to clarifying the content of other theoretical perspectives, exploring IT- and systemrelated concepts, and finding areas of overlap and potential synergy between traditional disciplinary silos.

\section{Background}

This paper defines a theoretical perspective related to a body of subject matter as an assemblage of concepts, assumptions, generalizations, associations, and methods that constitutes a useable viewpoint for recognizing, understanding, and analyzing situations and ideas within that body of subject matter. That view is consistent with Google Scholar searches on "theoretical perspective," which returned many thousands of hits identifying theoretical perspectives for many topics in many disciplines. In contrast, note that [3] treats theoretical perspectives as approaches for research design, e.g., variance, process, or systems perspectives.

A theoretical perspective is much more like a framework than like a theory. This paper's discussion of the FDTP follows distinctions between frameworks, theories, and models presented by the Nobel Prize winning economist Elinor Ostrom. As explained in [4, pp. 27-28] a framework helps to "identify the elements (and the relationships among these elements) that one needs to consider. ... Frameworks provide a metatheoretic language that is necessary to talk about 
theories and that can be used to compare theories." Theories "enable the analyst to specify which components of a framework are relevant for certain kinds of questions and to make broad working assumptions about these elements."... "Models make precise assumptions about a limited set of parameters and variables. Multiple models are compatible with most theories." In making those distinctions, [4, p. 27] noted "considerable confusion" related to interchangeable use of the three terms because "what one scholar calls a framework others call a model or a theory." The view of frameworks, theories, and models in [4] differs from the view expressed in [5], whose five categories of theory combine various aspects of frameworks, theories, and models as defined in [4].

The development of the FDTP was inspired by curiosity about a non-IS topic, rather than by a literature review, gap analysis, or Delphi study. It was more in the spirit of what [6] calls arm-chair theorizing. A layman interest in physics led to wondering whether there might be useful analogies between the types of issues in physics (often the object of "physics envy" [7]) and IS, which has self-consciously discussed its own legitimacy as a discipline for decades (e.g., $[8,9,10])$. The development of the FDTP started with loose analogies between IS topics and physics topics that appear in a popularized accounts of progress toward producing a "theory of everything" covering the structure and behavior of matter from the subatomic level to the cosmic level (e.g., $[11,12])$. The FDTP gradually emerged from numerous iterations of bouncing back and forth between ideas and issues in physics and in IS with the goal of distilling issues down to general terms rather than in disciplinespecific terms. For example, both a theory of everything in physics and a full understanding of IS call for identifying and describing forces, interactions, uncertainties, and so on. Perhaps fundamental forces apply to systems in organizations -- but what might they be? Perhaps something analogous to the Heisenberg uncertainty principle could apply to IS. Many initial ideas were rejected or modified through numerous iterations of asking questions such as "is this topic in physics somewhat analogous to interactions or overlaps or some other topic that might be included in the FDTP?" Despite this use of rough analogies between topics in IS and in physics, it would be silly to make too much of any imagined similarity between ideas in IS and ideas describing the behavior of subatomic particles. The key question is about whether the FDTP provides a plausible way to describe theoretical perspectives.

\section{Framework for Describing Theoretical Perspectives}

The current version of the FDTP consists of 25 concepts organized into seven categories: justification, coverage, focal points, attributes of entities, change, generalizations, and fundamental limitations.

- Justification: 1) rationale

- Coverage: 2) domain. 3) omissions

- Focal points: 4) primary entity types, 5) special cases of entity types, 6) facets of entities 7) portrayals of entities 8) functions of entities, 9) interactions of entities, 10) overlaps of entities.

- Attributes of entities: 11) characteristics, 12) performance variables, 13) phenomena

- $\quad$ Change: 14) events, 15) trajectories of change, 16) forces

- Generalizations: 17) axioms, 18) design principles, 19) frameworks, 20) theories, 21) models, 22) metamodels, 23) methods

- Fundamental limitations. 24) uncertainties, 25) indeterminacies.

The initial concepts such as rationale, domain, primary entity types, and omissions should seem obvious to most researchers, even though the domain and omissions for theories listed in the "Theories Used in IS Research Wiki" [13] are often unclear. Other concepts such as forces, interactions, overlaps, and uncertainties are not as obvious, and are not mentioned at all in many accounts of IS research results.

The concepts in the framework identify different topics but are not totally independent of each other. For example, trajectories of change and events are treated as separate concepts even though trajectories of change are sequences of events. Similarly, some interactions of entities involve overlaps of those entities. Overall, usefulness for thinking about systems seemed a more important criterion than mutual independence in deciding which concepts to include.

Note that this paper makes no claim that the proposed FDTP is the best possible FDTP. The application of the proposed FDTP to the WSP illustrates how it can help in clarifying the content of a theoretical perspective. Other possible FDTPs might help in other ways.

\section{The Work System Perspective}

This section provides background for Section 5, which demonstrates the potential usefulness of the FDTP by applying it to the WSP, which is often interpreted in teaching or research as little more than using the idea of work system to think about a situation or using the work system framework $[14,15]$ as a lens.

The WSP has developed over many years, as has happened with broadly applicable perspectives that are recognized more widely, e.g., general systems theory $[16,17]$, sociotechnical design $[18,19]$ and servicedominant logic [20, 21, 22]. The development of the WSP started with an attempt to develop a systems 
analysis method for business professionals, which was articulated as the work system method (WSM). The ideas underlying WSM were formalized as work system theory (WST). Subsequent developments related to service systems, workarounds, design principles, and other topics have been viewed as extensions of WST.

At this stage in its development, the core of WSP is work system theory (WST), which applies equally to WSs in general and to ISs. WST's three components are the definition of WS, the work system framework, and work system life cycle model. Since ideas related to WST and WSM have been presented many times, this section will focus on key points that minimize misunderstanding of the entire approach.

Definition of work. The WSP assumes that work is the application of human, informational, physical, and other resources to produce product/services for internal or external customers. Work can occur in businesses, governments, homes, and other situations where resources are used purposefully to produce outcomes.

Definition of WS. A work system is a system in which human participants and/or machines perform work (processes and activities) using information, technology, and other resources to produce specific product/services for internal and/or external customers [14]. The first and/or addresses trends toward automation of work by saying that work systems may be sociotechnical (with human participants doing some of the work) or totally automated.

Special cases. An IS is a WS most of whose activities are devoted to capturing, transmitting, storing, retrieving, deleting, manipulating, and/or displaying information $[14,15]$. This definition differs from 20 previous definitions in [14] and was one of 34 definitions of IS noted in [23]. It is also quite different from defining an IS as a representation [24, 25] or as a tool that is "used." Other important special cases include projects, service systems, self-service systems, and some supply chains (interorganizational WSs). For example, software development projects and other projects are WSs designed to produce specific product/services and then go out of existence.

Work system framework: a basic understanding of a WS. The nine elements of the WS framework are the elements of a basic understanding of a WS's form, function, and environment during a period when it is stable enough to retain its identity even though incremental changes may occur, such as minor personnel substitutions or technology upgrades. Those elements include customers, product/services, processes and activities, participants, information, technologies, environment, infrastructure, and strategies.

Work system life cycle model (WSLC): how WSs change over time. ISs and other WSs evolve through a combination of planned change through projects and unplanned change via adaptations and workarounds. The four phases in the WSLC (initial, development, implementation, and operation and maintenance) may be performed in many different ways. Typical activities and responsibilities associated with specific phases (e.g., designing, debugging, training, etc.) apply for waterfall, agile, prototyping, use of off-the-shelf applications, and shadow IT, even when several phases overlap or are combined through short iterations.

\section{Applying the FDTP to the WSP}

This section defines each of the FDTP's 25 concepts, thereby demonstrating its relevance to understanding the WSP. It omits details of many ideas related to WSP and the related references because that would absorb too much of this paper's 10 page maximum length.

Consistent with the way the FDTP serves as a framework (in terms of the discussion of frameworks, theories, and models in Section 2), it is not surprising that some parts of the FDTP are more important than other parts for describing the WSP. Recall how Ostrom's [4] distinction between frameworks, theories, and models implies that there is no reason to believe that every theory or model based on a framework needs to use every part of the framework (just as a model that uses BPMN does not need to use all of its constructs).

1. Rationale. (the reason for choosing a specific theoretical perspective). The WSP is an appropriate perspective for understanding and analyzing IT-enabled systems in organizations because the definition of WS implies that ISs and projects are WSs and therefore that the WSP covers central IS topics. WSs can be sociotechnical (with human participants) or totally automated (machines do all of the work in the WS). IS is a special case of WS most of whose activities are devoted to capturing, transmitting, storing, retrieving, deleting, manipulating, and/or displaying information. Projects are WSs designed to cease to exist after producing specific results. Highly focused supply chains and business ecosystems can be viewed as WSs or groups of interacting WSs that cross enterprises.

2. Domain. (the set of entities to which a theoretical perspective applies). The domain of the WSP includes WSs of all types and sizes, including ISs, projects, and service systems of all types and sizes. The domain needs to be broader than IS per se to support meaningful analysis of IT-enabled systems such as package delivery systems that perform physical activities not involved with processing information.

3. Omissions. (possibly relevant topics that are treated as beyond a perspective's scope). The WSP is designed for understanding WSs, but it addresses some important topics only indirectly or not at all. It recognizes issues related to IS/IT organizations, IS/IT 
careers, culture, competition, and marketing, but treats those topics as secondary to WS operation and evolution over time. Similarly, it recognizes individual differences between people but is not fundamentally about those differences.

4. Primary entity types. (primary categories of entity types within a theoretical perspective's area of maximum relevance or usefulness). The WSP is most relevant to the operation and/or development of ITenabled WSs. It is less useful at small scale for describing micro-activities within process steps and at large scale for describing the operation or evolution of an entire enterprise. It does not apply to static representations such as algorithms that are not activity systems.

5. Special cases of primary entity types. The most important split in the WSP is between sociotechnical WSs (where human participants perform some WS activities) vs. totally automated WSs (where machines perform all WS activities). Special cases of WS such as ISs, projects, and service systems may be sociotechnical or totally automated. Each special case can have its own special cases. All of the following are examples that inherit properties from WS in general and have additional inheritance relations of their own.

- Sociotechnical IS inherits from sociotechnical WS.

- IS project inherits from project.

- Open-source software development project inherits from software development project.

6. Facets of entities. (alternative faces or aspects of an entity that can be observed or analyzed). The idea of "facet" is like a facet of a cut diamond. It is not a separate component of the diamond, but rather a face or aspect that can be observed or analyzed. As an example, Table 1 lists 18 "facets of work" that can be viewed as facets of the processes and activities in a work system. Each facet applies to both sociotechnical and totally automated systems, is associated with specific concepts, brings evaluation criteria and design trade-offs, has sub-facets, and brings open-ended questions for starting conversations [26]. Some facets overlap (e.g., making decisions often involves communication). Inclusion of each facet is based on association with sets of useful concepts, evaluation criteria, and design trade-offs.

Table 1.18 facets of work

\begin{tabular}{|c|c|c|}
\hline $\begin{array}{ll}\text { - } & \text { Making } \\
\text { decisions } \\
\text { - } & \text { Communicating } \\
\text { - } & \text { Providing } \\
\text { information } \\
\text { - Representing } \\
\text { reality } \\
\text { - Applying } \\
\text { knowledge } \\
\text { - Thinking }\end{array}$ & $\begin{array}{ll}\text { - } & \text { Learning } \\
\text { - } & \text { Planning } \\
& \text { extrolling } \\
\text { - } & \text { Coordinating } \\
\text { - } & \text { Improvising } \\
\text { - } & \text { Processing } \\
& \text { information } \\
\text { - } & \text { Performing } \\
& \text { physical work }\end{array}$ & $\begin{array}{ll}\text { - } & \text { Performing } \\
& \text { support work } \\
\text { - } & \text { Interacting } \\
\text { socially } \\
\text { - } & \text { Providing } \\
\text { service } \\
\text { - } & \text { Creating } \\
\text { value } \\
\text { - } \\
\text { Maintaining } \\
\text { security }\end{array}$ \\
\hline
\end{tabular}

7. Portrayals of entities. (alternative ideas for visualizing the entirety of a WS or WS element). Many alternative portrayals apply to WSs in general and to elements of the WS framework. Examples include:

- customers as recipients of product/services vs. as beneficiaries of product/services vs. as people or other entities that pay for product/services

- product/services as outputs that are delivered vs. as results of extensive collaboration

- processes as idealizations of how work should be done vs. as descriptions of how work is executed

- participants as people with human needs and interests vs. participants as WS components

- participants as people performing actor roles in WSs vs. as users of technology

- information as machine-processed digital objects vs. as a conveyor of meanings that inform people

- technologies as tools used by users who perform work vs. as automated services that perform work.

8. Functions of entities. (functions that entities perform for the benefit of other entities). WSs may perform a wide range of functions for other entities. Thinking about ISs in relation to their functions is a departure from representation theory [24, 25] which views ISs as representations of real world systems. All of the following functions performed by ISs use representations, but are not about representation:

- providing access to information,

- defining and enforcing rules for collecting or sharing information,

- providing methods for aggregating information,

- providing methods for analyzing information,

- controlling activity sequences in workflows,

- enforcing compliance with business rules,

- creating alarms when predefined conditions occur,

- controlling or facilitating coordination,

- suggesting decisions,

- triggering automated functions,

- performing totally automated tasks autonomously.

9. Interactions. (unidirectional, mutual, or reciprocal actions, effects, relationships, influences, or interplay between two or more entities). Interactions between WSs are essential for the operation of any enterprise, organization, business ecosystem, or ITenabled WS. Interactions also bring significant risks, especially when interactions deviate from expectations. Various extensions of WST provide ideas that describe aspects of interactions involving WSs, such as a service value chain framework, a set of system interaction patterns, and a system interaction theory.

10. Overlaps between entities. (sharing of all or part of specific constituents or their components by two or 
more entities). Overlaps between WSs often play important roles in their operation, as when many ISs serve as integral components of other IT-enabled WSs that may or may not be ISs. For example, a package tracking IS is an essential component of a logistics firm's WS of moving packages. In other cases, an IS uses or includes another IS. An example is a sociotechnical accounting IS in which accountants decide how specific transactions and assets will be handled for tax purposes and then produce monthly or yearend financial statements. This is an IS because its activities are devoted to processing information. It is also supported by a totally automated IS that performs calculations and generates reports. In both the logistics and accounting cases, an IS that is an integral part of another WS cannot be analyzed, designed, or improved thoughtfully without considering how IS changes affect the WS being supported.

Different forms of overlap between WSs include separation or minimal overlap, significant overlap, and enclosure of one WS by another WS. With separation or minimal overlap, an IS provides information to a WS but is largely or totally separate from it, e.g., an IS that provides current medical knowledge to doctors. Most of that IS involves compiling information that doctors may access in their work. In other situations, the WS and IS overlap significantly, as when WS participants devote substantial attention and effort to activities in the IS. An important example related to medicine is how physicians multi-task while participating in two separate WSs simultaneously, a WS that provides medical care and an IS that provides and collects information. In yet other situations, an IS can operate completely within a WS, as when anesthesiologists use a real time IS for monitoring patient status during operations or when a factory's dispatching system helps in prioritizing work to be done in the factory.

11. Characteristics. (concepts used for describing or analyzing entities or their constituents). For example, characteristics of a WS as a whole include scalability, flexibility, resilience, degree of centralization, and fragility. Characteristics of processes and activities include degree of structure, complexity, integration, and rhythm. Characteristics of information include precision, age, timeliness, security, and bias.

12. Performance variables. (concepts used for describing or analyzing how well entities or their constituents operate). Performance variables for a WS as a whole include cost of operation and reliability. Performance variables for customers include customer satisfaction and a customer's total cost of d obtaining and using a WS's product/services. Note that goals are aspirations for a level of performance using a specific metric. Goals are joint attributes of stakeholders who set goals and a WS or WS element that ideally should perform well with regard to those goals.
13. Phenomena. (concepts identifying perceptible circumstances or occurrences that have an impact or are otherwise noteworthy but that are not components of a WS's structure or operation and are not inherent characteristics or performance variables for a WS or its elements). Phenomena related to a WS as a whole include digitalization, division of labor, and absorptive capacity. Phenomena related to participants include techno-stress, burnout, and information overload. Phenomena related to information include missing data and unintentional bias.

14. Events. (changes or actions that occur at a specific time or over a specific time interval). Important types of beneficial or harmful changes and actions within the WSP include:

- activities performed within the structure, capabilities, and purposes of a WS or several WSs

- modifications of elements of a WS that are produced through some version of the planned change sequence in the WSLC

- activities performed based on adaptations or workarounds (unplanned change) that may conflict with a WS's structure, capabilities, and purposes

- unplanned or accidental activities or events that degrade, disable, or destroy WS capabilities.

15. Trajectories of change. (identifiable sequences of changes or actions within a theoretical perspective). The WSLC summarizes a trajectory of planned change encompassing an initiation phase, development phase, and implementation phase leading to operation of a new or improved WS. Variations on the WSLC apply to situations where agile software development and/or DevOps approaches are used. Unplanned changes such as workarounds and adaptations can be associated with any of the four phases. A theory of workarounds summarizes a trajectory through which workarounds are imagined and implemented [27].

16. Forces. (influences of an entity or group of entities that induce or impede specific types of transitions in the state of other entities). Five types of forces apply to WSs as a whole.

- Cohesive forces tend to hold WSs together, e.g., incentives, goals, controls, alignment.

- Disruptive forces tend to make WSs less organized and may degrade them, e.g., misalignments, discontent, poor management, design flaws.

- Innovative forces encourage changes in WS architecture or operation based on benefits for customers, participants, or other stakeholders.

- Inertial forces resist planned or unplanned changes in WS operation. WSs often exhibit inertia by resisting transitions even when those changes may seem beneficial. 
- Forces from a distance (analogous to gravity) include economics, competition, regulation, technological change, and demographics.

Other forces operate as drivers or obstacles to WS change and are directly related to specific elements of the WS framework [28, p. 8].

17. Axioms. (statements assumed to be true for all WSs or for all WSs of a special type). WS axioms have been proposed but not tested extensively (e.g., [29], whose service system axioms apply equally to WSs).

18. Design principles. (statements that express desired characteristics of designed entities within a domain). Design principles may apply to all WSs within a domain, to specific types of WS within the domain, and/or to WSs associated with a community of practice. Unlike axioms, design principles often have exceptions, may be mutually inconsistent, and may conflict in practice. For example, as noted in [30], in some cases the principle "please the customers" may conflict with "do the work efficiently."

19. Frameworks. (organized sets of ideas whose relationships can be used to understand situations within a domain). [4] would say that WST and two of its main components are actually frameworks. An extension of WST that is a framework is the service value chain framework, which was designed to bring an explicit focus on service to discussions of WSs and ISs. It can be used with or without the work system framework. It stresses responsibilities of providers and customers, provider-customer interactions, common activities in service provision (set-up, service request, fulfillment, follow-up), and the extent of mutual visibility by providers and customers [31].

20. Theories. (based on [4], broad working assumptions about elements of a framework that are relevant for certain kinds of questions). One of the extensions of WST is a theory of workarounds [27, p. 1056]. Also, a currently unpublished theory of IS user satisfaction says, "The primary driver of an individual user's satisfaction with an IS that supports a WS is the degree to which the IS contributes to that user's efficiency in executing responsibilities within the WS and effectiveness in serving the WS's customers."

21. Models. (precise assumptions about a limited set of parameters and variables within a framework). Several extensions of the WSP can be viewed as models. An example is a model that identifies drivers and obstacles to IS innovation related to different elements of the work system framework [28]. Another is the organization of a proposed IS body of knowledge discussed in [32].

22. Metamodels. (models identifying concepts and relationships that can be used for modeling specific situations.) A series of metamodels was produced that tried to address limitations of the work system framework. Those metamodels restate every element of the work system framework in a more detailed form that shows relationships through which WSs operate and serve their customers. Thus far they have been used mostly for identifying potentially useful analysis tools that can be used with WSM.

23. Methods. The work system method (WSM) coevolved with WST. It is a semi-formal SA\&D approach designed to help business professionals visualize WSs in their own organizations and collaborate more effectively with IS/IT professionals. During 2003-2017, individual students or teams (mostly employed MBA and Executive MBA students) used various versions of WSM to produce over 700 management briefings recommending improvements of problematic IT-enabled WSs, mostly in their own firms (e.g., [33]).

24. Uncertainties. (extent of incompleteness or inaccuracy of information about the past, current, or future states, events, or of causes of states or events related to entities or phenomena). Differing degrees of uncertainty apply to how a process or activity will be executed, to the quality of information, to how well technology will operate, to the skills and seriousness of WS participants, and to the exact form and quality of specific product/services.

25. Indeterminacies. (fundamental limitations on the possibility of knowing specific aspects of the past, current, or future states, events, or causes related to entities or phenomena). At some level of detail, it is impossible to produce replicable, non-subjective explanations of how and why WS events occur or have occurred in the past, especially when information about participant intentions and other important factors are not observed or recorded.

\section{Using the FDTP to Apply the WSP to Digital Transformation}

Building on the illustration that the FDTP can be used to describe a theoretical framework (in this instance, the WSP). this section illustrates an application of the FDTP for clarifying an important concept. It shows how the FDTP can help in applying the WSP to DT. A recent literature review [34] found 282 articles on that topic, of which only 28 actually defined DT. Many of those definitions had significant shortcomings including conflation between the concept and its impacts, circularity, lack of parsimony, and use of unclear terms. None of the DT definitions listed in [34, p. 3] viewed DT as creating or improving work systems. Thus, applying the WSP to DT might help in describing and understanding DT.

The following discussion applies the FDTP to address a challenge presented by a JAIS Special Issue that is designed to advance theoretical diversity in IS by 
featuring diverse predictive theories related to DT [35]. The FDTP is used here to outline a framework of ideas related to the WSP that lead to a proposed DT success theory (DTST) that is designed to help executives manage a DT initiative through to its completion. The DTST is mentioned as item \#20 in the following discussion. This section does not try to explain or justify the DTST, which would require a full paper.

1. Rationale for applying WSP to DT. A WSPbased approach to DT is appropriate because enterprises serve their customers through the operation and interactions of numerous WSs. DT is defined here as an enterprise-level transformation in which ICT is an essential component of strategically significant changes in mission-critical WSs. A DT occurs through a DT initiative, which is defined as an identifiable enterpriselevel initiative involving the initiation, oversight, and management of one or more DT-related projects from inception to completion or abandonment. DT initiatives that ignore the WS level or aggregations of WSs likely will miss important operational topics and issues.

2. Domain of DT. A WSP-based view treats DT as an enterprise-level transformation as defined above. DT is viewed as purposeful and project-oriented. A WSPbased view of a DT initiative focuses on selected mission-critical WSs, not on the entire enterprise, and not just on upgrading shared IT infrastructure.

3. Omissions from the WSP view of DT. A WSPbased view of DT is not about ICT, not about the diffusion or progress of technology, not about some vague notion of "disruption," and not about broader industrial or societal changes that are sometimes seen as the essence of DT.

4. Primary entity types. These are mission-critical WSs and projects attempting to improve those WSs or create different WSs.

5. Special cases of DT. The WSP-based view of DT can be applied to different types of DT initiatives, although there is no obvious hierarchy of categories. It might be interesting to try to understand distinct aspects of DT initiatives related to operational excellence vs. product/services vs. customer experience. Another potentially interesting set of categories involves the size and form of the enterprise. For example, DT initiatives within small to medium enterprises probably differ in many ways from DT initiatives in large multinational enterprises or in supply chains or ecosystems that cross enterprises.

6. Facets of DT. The idea of facets raises many questions about potentially interesting facets of DT that are not described as steps in a DT initiative. For example, facets of work such as making decisions, communicating, coordinating, controlling execution, and maintaining security all apply across DT initiatives. It is possible that those and other facets of work appear in different forms as DT initiatives unfold. Facets specifically related to DT itself include technology choices, organizational change, competitive response, and response to resistance.

7. Portrayals of DT. A WSP-based approach tends to focus on DT goals and on progress in achieving intended changes in mission-critical WSs. Other bigpicture portrayals of DT (mentioned previously under \#3, omissions) would focus more on technological change, diffusion of technology, disruption, social change, and so on. It would be interesting to observe ways in which DT projects address different portrayals of specific WS elements. For example, to what extent do DT projects treat customers as recipients of product/services vs. as beneficiaries of product/services vs. as people or other entities that pay for product/services? To what extent do they treat WS participants as people with human needs and interests vs. as WS components performing actor roles? To what extent do they treat information as digital objects processed by machines vs. as signs conveying meanings that inform people?

8. Functions of WSs that are affected by DT. A WSP-based view of DT should attend to any necessary changes in functions that WSs perform to support other WSs within an enterprise and to provide product/services for customers. As noted in the earlier discussion of the WSP, typical examples of those functions go from providing access to information and defining and enforcing information-driven rules through suggesting decisions and automatic execution of totally automated tasks. Empirical research about DT might identify the relative frequency of modifying different types of WS functions in DT initiatives.

9. Interactions related to DT. DT-related projects should be careful about interactions between missioncritical WSs because changes in individual WSs are likely to affect interactions between some of those WSs.

10. Overlaps affected by DT initiatives. DT initiatives that try to increase efficiency and effectiveness by changing the structure of organizations likely involve changes in the extent of overlap between mission-critical WSs. The related tradeoffs involve the relative advantages of overlap between WSs for focusing responsibility and minimizing handoffs versus separation between WSs to maximize clarity and reliability and to minimize risks related to multitasking.

11. Characteristics related to DT initiatives. Almost any important characteristic of mission-critical WSs might be affected by changes related to a DT initiative. Also relevant to DT initiatives are both stable project characteristics and transient project characteristics that change as DT initiatives unfold.

12. Performance variables affected by DT. A DT initiative might affect future results related to any important performance variable related to a WS as a whole or to any WS element. The DTST in item \#20 
applies "favorability ratings" of various "resources for change" related to the progress or status of a DT initiative. By analogy with the commonly used resourcebased view (RBV), the favorability of many different types of "resources for change" affect the likelihood that DT initiatives will achieve their goals. In effect, those favorability ratings are performance variables describing specific aspects of the current state of a DT initiative at any point during its continuance. The DTST (item \#20) is based on the favorability of five types of resources for change. Largely tangible resources include (1) momentum toward the DT initiative's transformational vision and (2) capabilities needed to achieve that vision. Intangible resources include (3) forces affecting the entirety of a work system, (4) drivers and impediments related to specific WS elements, and (5) catalysts affecting the micro-dynamics of DT changes. Focusing on the five types of resources for change is an attempt to go beyond frequently mentioned success factors and risk factors, such as the extent of executive support and involvement, the degree of prior experience related to similar projects, the availability of resources, and the level of agreement about goals and methods.

13. Phenomena related to DT. Almost any important phenomenon related to an entire WS or a WS element might be affected by a DT initiative. Other phenomena are linked more closely to the nature of DT. An example is a WS's DT-related momentum, i.e., the extent to which its current state and critical path to completion are aligned with the DT's transformational vision. Another phenomenon is the impact of catalysts that affect the micro-dynamics of DT. Three of those catalysts are needing, understanding, and liking the changes, all of which involve cooperation vs. resistance of WS participants and other stakeholders [28].

14. Events that occur during DT initiatives. The start and completion of every step in a DT project can be viewed as a relevant event that contributes to the completion of the DT initiative. Also, many DT initiatives change the timing, nature, or other aspects of operational events that occur as mission-critical WSs perform processes and activities.

15. Trajectories of change. The planned trajectory of an ongoing DT initiative may be modified based on the current status of on-going projects and assessments of future issues and possibilities. The phases of DT can be viewed as a 5-phase variation on the WSLC that nonetheless recognizes that iterations and overlaps between phases may occur:

1) Impetus: recognition of enterprise-level challenges that a DT could try to address.

2) Transformational vision: management vision of how those challenges will be addressed

3) Resource acquisition: the process of creating, obtaining, improving, and/or channeling resources that are needed for DT implementation or will help with implementation

4) Implementation: deploying or exploiting resources and making other WS changes needed to achieve the transformational vision

5) Operation and maintenance: ongoing operation and support of the transformed WSs.

16. Forces that affect DT. All five types of forces that were mentioned in relation to the WSP may have positive or negative impacts on DT-related changes in a WS. Cohesive forces and innovative forces aligned with the transformational vision tend to propel the progress of DT initiatives. The five alignment arrows within the work system framework [15] highlight typical paths for cohesive forces. Disruptive forces and inertia tend to impede progress. Various forces at a distance (analogous to gravity) also may propel or impede progress toward DT goals. For example, competitive pressures may propel that progress in a specific case whereas regulatory rulings may impede that progress.

17. Axioms related to DT. It is unclear whether nontrivial axioms related to DT initiatives themselves can be produced. Previously mentioned axioms related to WSs would apply to the WSs that are objects of the DT initiative, but it is not clear whether those axioms would provide any useful insights.

18. Design principles related to DT. Much of the advice about DT that consultants discuss in webinars and publish in white papers can be viewed as design principles related to the form and operation of DT initiatives. Most of those design principles seem to be based on experience and knowledge about business. Design principles related to sociotechnical WSs such as those in [30] and elsewhere might help in designing WS improvements.

19. Frameworks related to DT. Recall the view in [4] that frameworks "identify the elements (and the relationships among these elements) that one needs to consider." In essence, the DT discussion thus far has presented a framework that supports the DTST presented in item \#20. That framework includes the WSP itself, the definition of DT and DT initiative, and the DT-related ideas explained thus far in items \#1 through \#18

20. Theories related to DT. The proposed DTST (used here as an illustration of a theory related to DT) is stated from the viewpoint of executives and managers who launch a DT initiative, monitor it, and engage in an ongoing process of making decisions that try to maximize its chances of success. Those decisions need to consider positive and negative aspects of five types of "resources for change" that may be relevant to a DT initiative. Throughout the DT initiative, executives need to make sense of a wide array of information that ranges from largely objective (e.g., the degree of completion of a specific step in a project or the availability of specific ICT resources) through highly subjective (e.g., the level of anger and resistance among stakeholders who will be 
affected adversely or the level of external threat such as a likely change in governmental regulations). A DTST may help them relate a plethora of diverse information to likely outcomes of the DT initiative based on the current situation at any time from before launching the initiative through near its conclusion.

The proposed DTST states: At any time during a DT initiative, the favorability of five categories of resources for change for WSs targeted by the DT initiative contribute individually and in combination to the likelihood of the initiative's eventual degree of completion and business success. The categories are momentum, capabilities, forces, drivers/impediments related to WS elements, and catalysts. Each category includes resources for change whose state can be described at any time during a DT initiative and can be compared to an ideal state relative to the progress to date of the DT initiative. Favorability ratings for resources for change provide a way to organize that comparison.

The DTST itself is a predictive theory (as requested by [35]), but executives might prefer to use it mostly as a mental model for guiding their thinking as they monitor a DT initiative. They also can use it to organize information in a formal monitoring system that combines typical project management data with favorability ratings collected via periodic surveys, interviews, meetings, and other data collection means (in the spirit of an executive information system). Data inputs describing individual perceptions would have to be aggregated into favorability ratings by category, by WS, and possibly by business unit.

21. Models related to DT. Formal models would be needed if executives want numerical predictions or probability distributions of outcomes. Producing reliable prediction models based on favorability ratings seems like an extremely challenging problem. Just aggregating favorability ratings in a meaningful way could be challenging because an overall favorability rating would be based on weightings of less aggregated favorability ratings. It is unlikely that analysis of any existing dataset of past DT initiatives could provide parameters that could guide those weightings. Also, regardless of how good a model's predictions might seem, mergers or financial, regulatory, or competitive surprises could divert resources or change goals and might simply be showstoppers. Overall, the DTST seems more useful for organizing ideas and information and less useful for specifying a predictive model.

22. Metamodels related to DT. Metamodels related to the WSP might be useful in modeling specific WSs. They probably would not provide insights beyond the insights from the DT framework and DTST.

23. Methods for DT. It is difficult to imagine a single method that would apply across all of the situations in which DT initiatives might occur. Despite that, general steps or methods are implied by many articles and consulting company white papers that have discussed DT.
24. Uncertainties related to DT. The multitude of changes required by most DT initiatives implies high uncertainty about how those initiatives will unfold. It seems a reasonable guess (but only a guess) that many industry accounts of DT initiatives tend to exaggerate the extent to which the trajectories of those initiatives unfolded consistent with a plan publicized at the outset.

25. Indeterminacies for DT. As is true of both WSs in general and projects in general, there is no way to know in advance exactly what every participant in a DT initiative will try to do or will actually accomplish.

\section{Implications and conclusions}

This paper contributed to knowledge about IS by explaining and illustrating a way to delve deeply into the nature of theoretical perspectives and into key concepts within different theoretical perspectives. This paper presented the FDTP as a broadly applicable framework for clarifying the content of theoretical perspectives. It applied the FDTP to summarize the content of the WSP. Then it applied the FDTP to a WSP perspective on digital transformation, thereby illustrating broader applicability of ideas in the FDTP. This paper's discussion of the FDTP and its two applications of the FDTP could be extended further.

Improving the FDTP. The FDTP was developed through an informal, iterative process and could be developed further. Other researchers might pursue that goal using iterative methods mirroring those used here or might use completely different approaches. Experts in ontology, epistemology, or linguistics might be able to suggest other approaches to developing alternative FDTPs from scratch or approaches for improving the version presented here.

Applying the FDTP for understanding other theoretical perspectives. The FDTP could be tested by applying it to organize ideas in other theoretical perspectives related to systems in organizations, such as representation theory, general systems theory, sociotechnical systems theory, living systems theory, the viable systems model, soft systems methodology, activity theory, organizational routines, information theory, service-dominant logic, and so on. Comparisons of applications of the FDTP to different perspectives could generate useful insights about the advantages and limitations of using those perspectives.

Applying the FDTP for exploring important ITand system-related concepts. This paper's attempt to apply the FDTP to DT might be replicated to some extent by applying a current or future version to other concepts such as IS development, artificial intelligence, big data, affordances, IS usage, crowdsourcing, sociomateriality, absorptive capacity, and so on. 
Finding areas of overlap and potential synergy between traditional disciplinary silos. Applying the FDTP to some of the central theoretical perspectives or even major topics in a variety of system-related disciplines might find substantial overlaps or synergies between important ideas in those areas and ideas in the WSP or other theoretical perspectives mentioned above. System-related disciplines where this might be most successful include operations management, industrial engineering, marketing, organizational behavior, and service science. Overlaps or synergies with those disciplines might lead toward new attempts at integration or at least greater cooperation across parts of what are currently viewed as separate disciplines.

\section{References}

[1] D. Hovorka and B. Mueller, "knowing what we know: where to now?'HICSS 2021 Call for Papers.

[2] D. Hovorka and B. Mueller, "informing research: where to now?" HICSS 2022 Call for Papers.

[3] A. Burton-Jones, E. R., McLean, and E. Monod, "Theoretical perspectives in IS research: from variance and process to conceptual latitude and conceptual fit." European journal of information systems, 24(6), 2015, pp. 664-679.

[4] Ostrom, E., Understanding Institutional Diversity, Princeton University Press. 2009

[5] S. Gregor, "The nature of theory in information systems. MIS Quarterly, 30(3) 2006, pp. 611-642.

[6] V. Grover and K. Lyytinen, New state of play in information systems research. MIS Quarterly, 39(2), 2015, 271-296.

[7] Nelson, R.R., Physics envy: get over it. Issues in Science and Technology, 31(3), 2015, pp.71-78.

[8] I. Benbasat and R. Zmud, "The Identity Crisis Within the IS Discipline: Defining and Communicating the Discipline's Core Properties," MIS Quarterly 27(2), 2003 , pp. 183-194

[9] K. Lyytinen and J. L. King. "Nothing at the center?: Academic legitimacy in the information systems field." JAIS, 5(6), 2004, pp. 220-246

[10] K. K. Kautz, "Debate Section Editorial Note: Is Information Systems a Science?" CAIS, 43(1), 2018.

[11] D. Lincoln, The Theory of Everything: The quest to explain all reality: Course guidebook. The Teaching Company: Chantilly, Virginia, USA. 2017.

[12] N. Mee, Higgs force: The symmetry-breaking force that makes the world an interesting place. James Clarke \& Co., 2012.

[13] K. R. Larsen and D. Eargle, (Eds.) "Theories Used in IS Research Wiki. Retrieved from http://IS. TheorizeIt.org

[14] S. Alter, "Defining information systems as work systems: implications for the IS field," European Journal of Information Systems, 17(5), 2008, pp. 448-469

[15] S. Alter, "Work System Theory: Overview of Core Concepts, Extensions, and Challenges for the Future," Journal of the Association for Information Systems, 14(2), 2013, pp. 72-121.

[16] R. L. Ackoff "Towards a system of systems concepts. Management Science:” 17(11), 1971. pp. 661671.
[17] L. Skyttner, "General systems theory: origin and hallmarks." Kybernetes, 25(6), 1996, pp. 16-22.

[18] E. L. Trist, "The Evolution of Socio-Technical Systems: A Conceptual Framework and Action Research Program" Occasional Paper No. 2. Ontario, Canada: Ontario Quality of Working Life Centre, 1981.

[19] E. Mumford, "The story of socio-technical design: Reflections on its successes, failures and potential," Information Systems Journal, 16(4), 2006, pp. 317-342.

[20] S. L. Vargo and R. F. Lusch. "The four service marketing myths: remnants of a goods-based, manufacturing model." Journal of Service Research, 6(4), 2004, pp. 324-335

[21] S. L. Vargo and R.F Lusch, "Service-dominant logic: continuing the evolution." Journal of the Academy of Marketing Science, 36(1), 2008, pp.1-10.

[22] S. L. Vargo and R. F. Lusch, "Institutions and axioms: an extension and update of service-dominant logic," Journal of the Academy of Marketing Science 44(1), 2016, pp. 523

[23] S.K. Boell and D. Cecez-Kecmanovic, "What is an information system?" Proceedings of Hawaii International Conference on System Sciences, HICSS, 2015, pp. 4959-4968, IEEE.

[24] Y. Wand and R. Weber. "Towards a Theory of the Deep Structure of Information Systems," ICIS 1990.

[25] A. Burton-Jones, J. Recker, M. Indulska, P. Green, and R. Weber. "Assessing representation theory with a framework for pursuing success and failure." MIS Quarterly, 41(4), 2017, pp.1307-1333.

[26] S. Alter, "Facets of Work: Enriching the Description, Analysis, Design, and Evaluation of Systems in Organizations," Communications of the Association for Information Systems, 2021, in press.

[27] S. Alter, "Theory of Workarounds," Communications of the Association for Information Systems: 34( 55), 2014, pp. 1041-1066

[28] S. Alter, "A Systems Theory of IT Innovation, Adoption, and Adaptation," Proceedings of European Conference on Information Systems, ECIS 2018

[29] S. Alter "Service System Axioms that Accept Positive and Negative Outcomes and Impacts of Service Systems," Proceedings of ICIS 2017

[30] S. Alter. and Wright, R. "Validating work system principles for use in systems analysis and design." Proceedings of ICIS 2010.

[31] S. Alter. "Viewing systems as services: A fresh approach in the IS field. Communications of the Association for Information Systems, 26(11), 2010, pp. 195-224.

[32] S. Alter. "An Open-Ended Work System Knowledge Model for Visualizing, Organizing, and Accessing Knowledge about Information Systems in Organizational Settings," Proceedings of HICSS 2021.

[33] D. Truex, S. Alter, and C. Long, "Systems analysis for everyone else: Empowering business professionals through a systems analysis method that fits their needs." Proceedings of ECIS 2010.

[34] G. Vial, "Understanding digital transformation: A review and a research agenda". The Journal of Strategic Information Systems, 28(2), 2019, pp. 118-144.

[35] M. L. Markus and F. Rowe, "Guest Editorial: Theories of Digital Transformation: A Progress Report. Journal of the Association for Information Systems, 22(2), 2021, pp. 273-280. 